\title{
Web-based Implementation for Marine Casualty Information on Google Map
}

\author{
Seojeong Lee ${ }^{1}$, Hyoseung $\mathrm{Kim}^{1}$ and Hye-young $\mathrm{Kim}^{2}$ \\ ${ }^{1}$ Computer Engineering Department, Korea Maritime and Ocean University, 727 \\ Tajongro, Youngdo-gu, Busan, 606-791, Republic of Korea \\ ${ }^{2}$ Game Software Department, Hongik University, 2639 Sejong-ro, Jochiwon-eup, \\ Chungcheongnam-do, 339-701, Republic of Korea \\ sjlee@kmou.ac.kr,khs9962@gmail.com,hykim@hongik.ac.kr
}

\begin{abstract}
Safety navigation of vessels is always the most important issue in maritime field. Recently, according to increasing the use of electronic equipment onboard. the requests for safety related information services have been growing up. To sypport this, International Hydrographic Organization suggests the concepv of commonmaritime data structure. Since 2010, S-100 standard and relative productspecifications have been defined as a part of that concept, covering the electronic navigational chartdala land safe navigation related data. In this paper, we introduce an empirical application of S-100 standard, applied to marine casualty information. We analyze marne casualty cases reported in Korea for recent four years, make XML documents with schema design and then implement an application to present the marine casualty information on google map.
\end{abstract}

Keywords: Marine casualty information, S-100 standard, XML, web-based application

\section{Introduction}

In the most areas which are not relied on electronic equipment quite much, the capability of a person in charge is an important factor of the quality of work. Similarly, in the traditional vessel navigation using paper chart, the navigator's capability can make the voyage successful or not. Aecording to increasing the use of electronic equipment onboard and improving the network capability between onboard and shore side, navigators as well as shore side stakeholders have needed the better service for safe navigation. Only several years ago, there were abie to use just VHF and very expensive internet. Taking into account on this, IM0 (nternational Maritime Organization) has introduced the concept of e-navigation in 2006, which organization is a United Nations maritime agency to develop the regulations of technical issues for vessels, operational issues for navigational affairs and training issues for the mariners. The definition of e-navigation [1] is as follows:

"e-Navigation is the harmonized collection, integration, exchange, presentation and analysis of maritime information onboard and ashore by electronic means to enhance berth to berth navigation and related services, for safety and security at sea and protection of the marine environment"

To realize the harmonized service as mentioned on the definition of e-navigation, not only computing techniques but also standardized protocols of the information service. In the case of the techniques and protocols related to navigational charts, these should be standardized to 
provide consistent and exact services to navigational vessels. IHO (International Hydrographic Organization) has a role to develop the standards related to navigational chart, which is carried out in cooperation with IMO.

In 2009, the concept of CMDS (Common Maritime Data Structure) was defined as the data service protocol supporting the e-navigation. This means that a common structure would be designed to be use commonly, and then all the vessels which perform international voyages should comply with the structure in the near future. S-100 standard can be a starter to build the total common structure. It deals with the information of electronic navigational chart and the information related to safe navigation with registering product specifications. S-101 for electronic chart and S-102 for bathymetry have been registered so far. Various kinds of product specifications would be expected to be registered. These are specified as XML (eXtensible Markup Language) to be provided on the web [2].

This paper introduces an empirical application of S-100 standard, applíed to marine casualty information. The analysis of marine casualty cases reported in Korea for recent four years and the creation of XML documents with schema design are processed before implementing the application to present the maripe casualty information on google map.

\section{Related works}

\subsection{S-100 Standard}

IHO S-100 standard provides a contemporary hyorographic geospatial data standard that can support a wide variety of hydrographic-related digital data sources, aligned with the ISO 19100 series of geographic standards for the easier integration of hydrographic data and applications into geospatial solutions [2]. IHO S-100 standard contains how to define the information structure of electronic navigational charts and the related solutions as called a product specification. Figure 1 shows the relationship between S-100 and product specitication. If describes the exact number will be assigned when the product specification is regisfered.

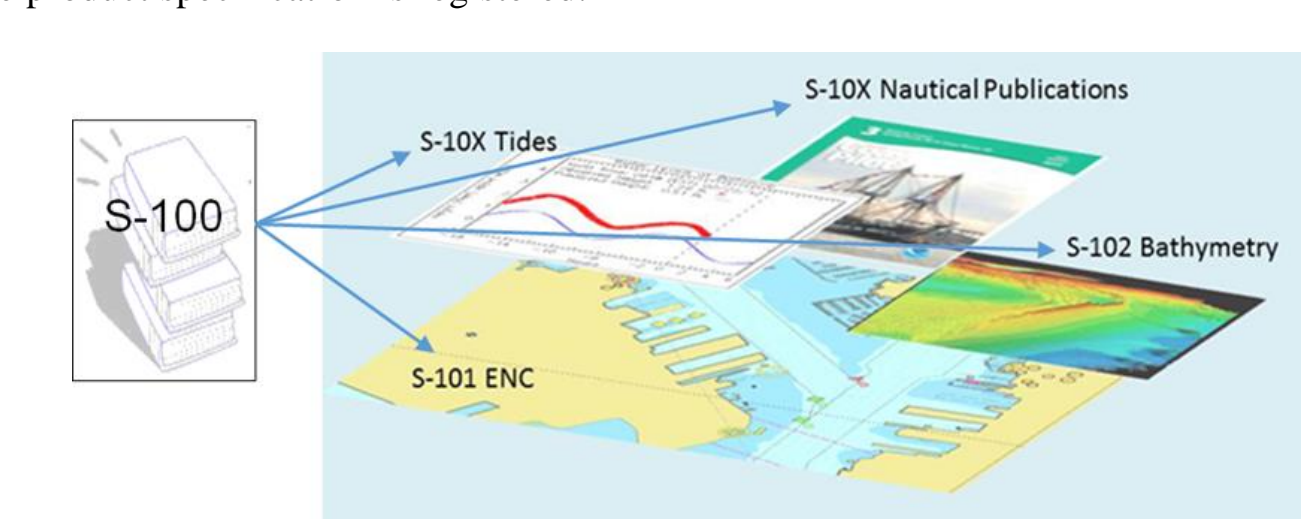

Figure 1. The Relationship between S-100 and Product Specifications

\subsection{Previous Works}

PPMS (Potentially Polluting Marine Sites) [3] is a research to provide the information of potentially polluting area to navigational vessels which are close to the reported area. After designing the schema of potentially polluting shipwrecks, dumping areas, pipelines and other pollution related information, PPMS geo-spatial database is created based on the schema. S-100 standard is considered to design the schema and to 
create database using UML (Unified Modeling Language) and GML (Geographic Markup Language).

ICEMAR project [4] funded by EC (European Commission) has been performed for three years from 2011. One of goals of this project is to develop S-100 based product specification for delivering sea ice information to vessels operating in icy waters on ECDIS (Electronic Chart Display and Information System) on the bridge.

\section{Analysis of Marine Casualty Information}

KMST (Korea Maritime Safety Tribunal) has published casebooks of marine casualty information, called "the Casebook of KMST" every year. The book offers the detail information about marine casualty cases which are held in Korean territorral waters. This paper analyzes the marine casualty cases for recent four years from 2009 using UML [5-8].

Figure 2 shows the UML class diagram as the analysis result of the dollision type of marine casualty cases. 'marine casualty information'class is conespondent to the 'ship' class which is for describing ship's condition, 'acctype' clas for accident type. Also, a 'marine casualty information' class is aggregated with 'weather' class which is describing for weather condition, 'judgment'class for the result of judgment about the casualty, 'date_time' class for exact date and time, socation' class for latitude and longitude and 'SEAARE' class for sea are.

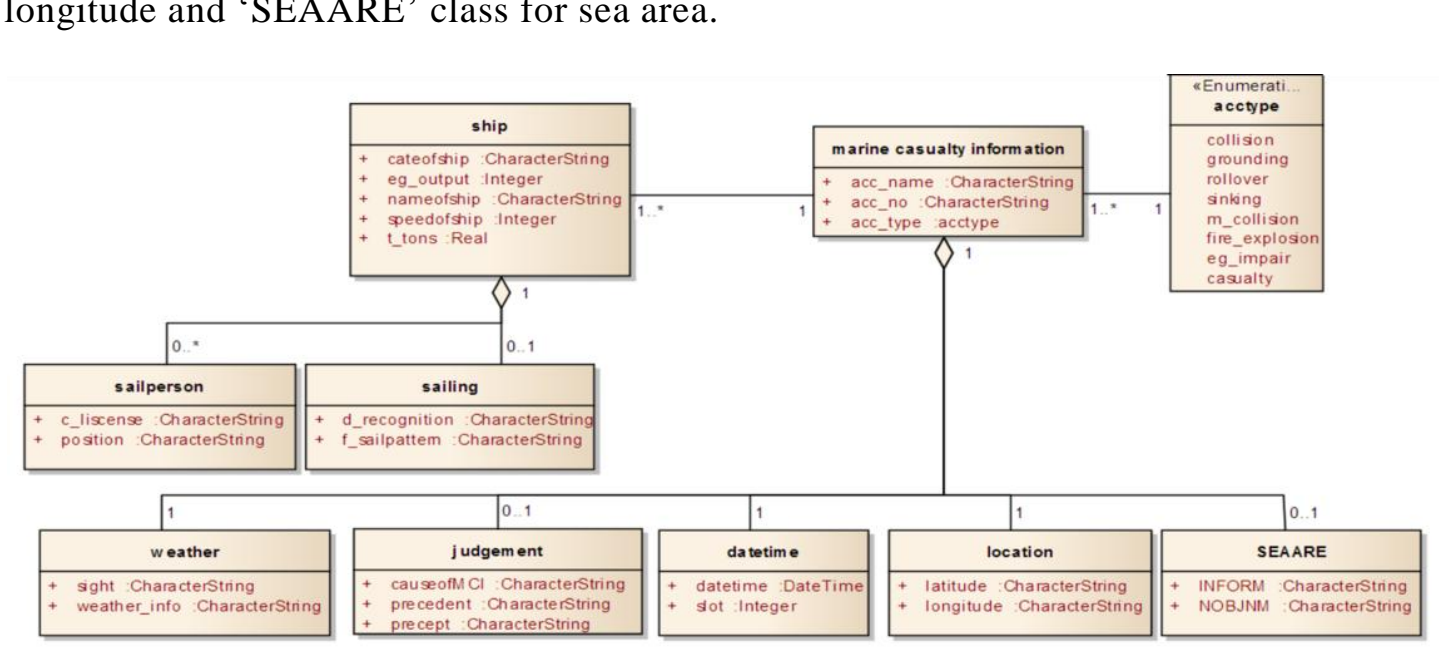

Figure 2. The UML Class Diagram for Marine Casualty Information

\section{Implementation of Marine Casualty Application}

This paper implements a web-based application to display the collision type of marine casualty information on google map. Followings are the steps for the implementation. In this paper, the design process of XML schema definition is not described in detail, however, Figure 3 is the XML schema definition translated from the design process.

a. Create XML schema definition from the design for the collision type of marine casualty information. Figure 3 shows the XML schema definition of this implementation.

b. Create XML documents of marine casualty information which is combined the schema definition to source data. 
c. Parse XML documents to identify position information such as the longitude value and the latitude value and the other part of casualty information to be displayed.

d. Display the graphic market on the position of casualties.

e. When users click or touch the specific market, the other part of casualty information is displayed using a small window.

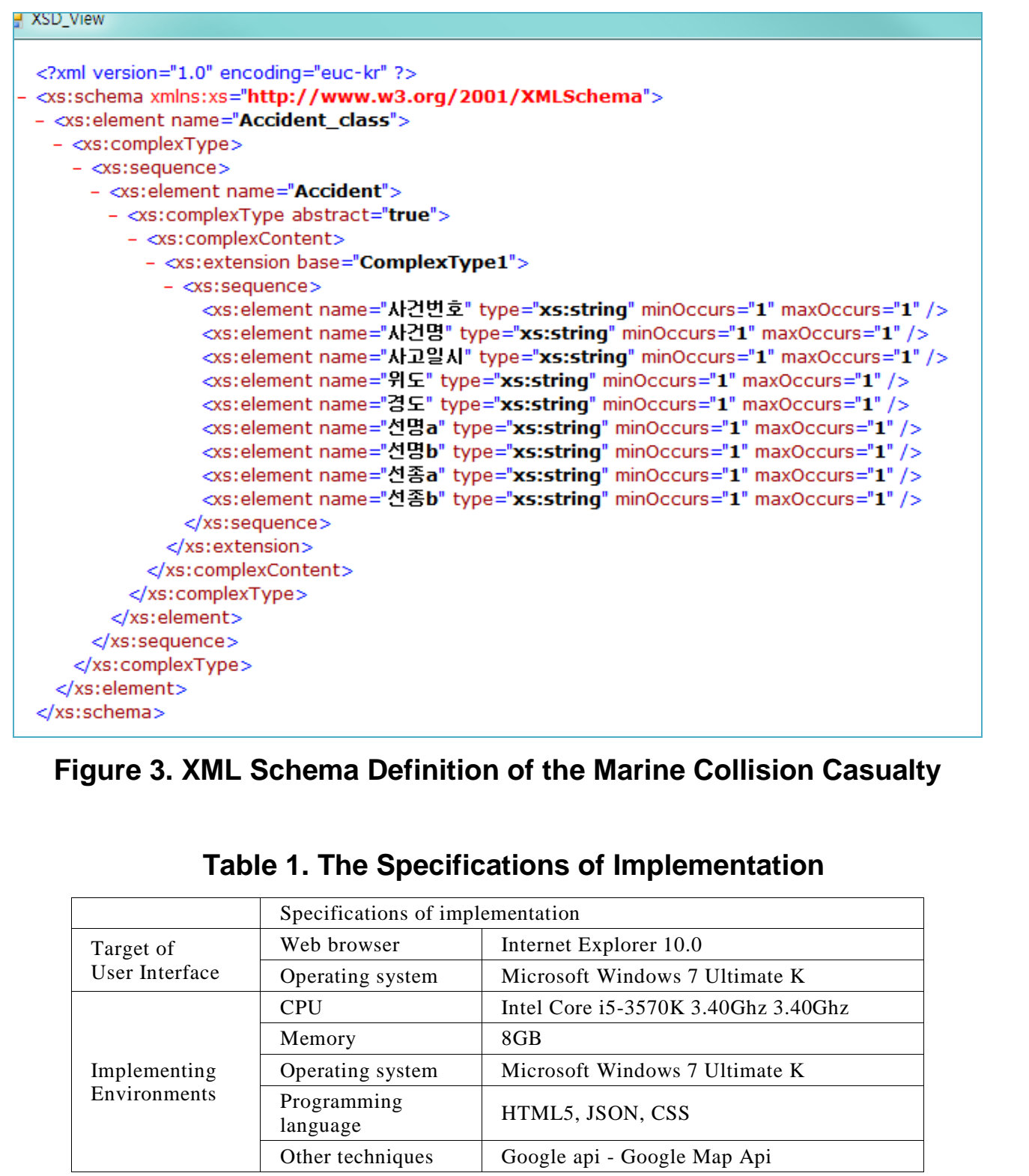

Table 1 shows the detail specification of implementation. The user interface environments are considered to be provided marine casualty information. Figure 4 shows a sample result of the web-based implementation displaying on google map. 


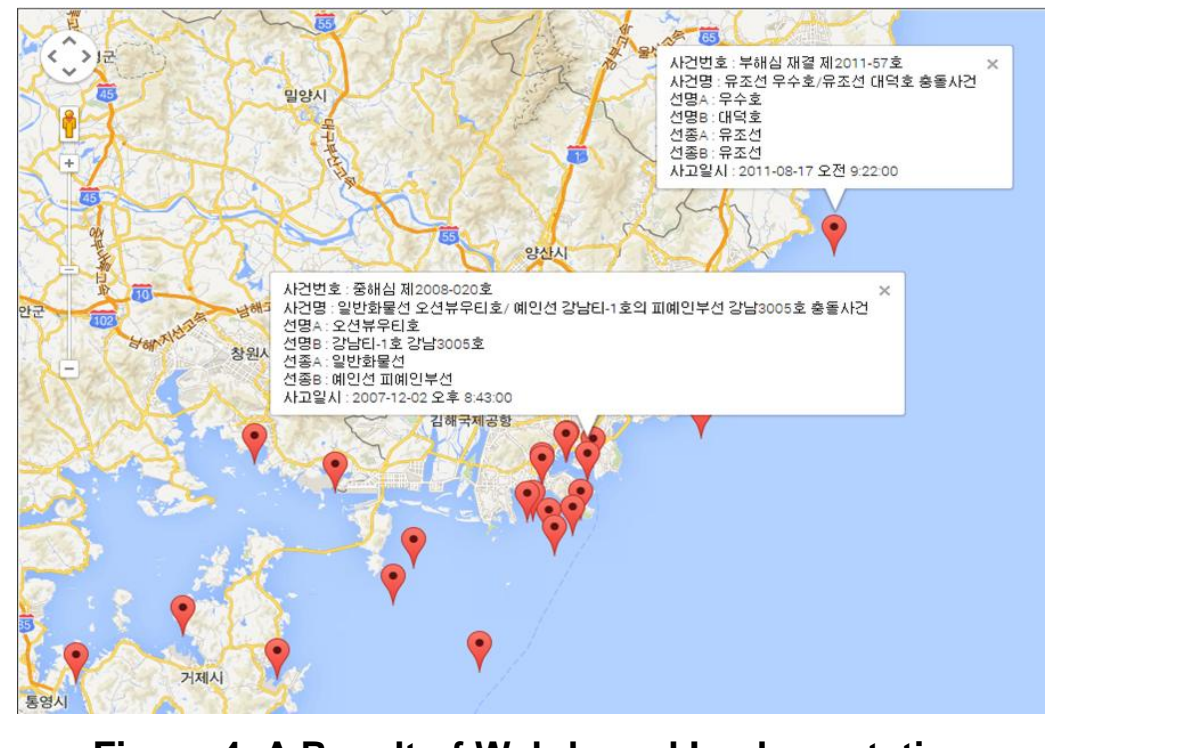

Figure 4. A Result of Web-based Implementation

\section{Conclusion}

Recently, as the IHO S-100 standard has been Assued to support common maritime data structure, the studies on various kinds of safe navigation related information have being increased. In this paper, we introduced an empirical application of S-100 standard, applied to marine casualty information. Wê performed the analysis of marine casualty cases reported in Korea forgecent four years and the creation of XML documents with schema design and the implementation of the web-based application to present the marine casualty information on goggle map.

\section{Acknowledgements}

This article is supported by KEtI (Korea Evaluation Institute for Industrial Technology).

\section{References}

[1] International/Maritime Organization, MSC 85/26/Add.1, Annex 20 - Strategy for the development and implementation of e-navigation, IMO, (2008) Nov.25-Dec.6; London, United Kingdom.

[2] International Hydrographical Organization, Special Publication No.57. Ed. 3.1-IHO Transfer Standard for Digital Hydrographic Data, IHO, Monaco, (2000).

[3] M. Giuseppe, C. Brian and A. Lee, "Developing a GIS-Database and Risk Index for Potentially Polluting Marine Sites", Proceedings of 2012 Canadian Hydrographic Conference, (2012) May 1517, Niagara Falls, Ontario, Canada.

[4] German Ice Service (BSH), HSSC5-07.9A-Development of the S-100 based Ice Information Product, IHO Hydrographic Services and Standards Committee (HSSC), (2013) November 11-13; Shanghai, China.

[5] Korea Maritime Safety Tribunal, The casebook of KMST in 2008, KMST, Seoul, (2009).

[6] Korea Maritime Safety Tribunal, The casebook of KMST in 2009, KMST, Seoul, (2010).

[7] Korea Maritime Safety Tribunal, The casebook of KMST in 2010, KMST, Seoul, (2011).

[8] Korea Maritime Safety Tribunal, The casebook of KMST in 2011, KMST, Seoul, (2012). 


\section{Authors}

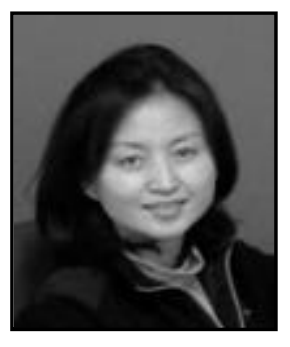

Seojeong Lee, She works for Korea Maritime and Ocean University as an associate professor. She is interested in software development and quality issues related to vessel navigation and communication systems. The theme of her Ph.D. dissertation from Sookmyung Women's University in 1998 was a software development methodology. After joining to Korea Maritime and Ocean University in 2005, she has tried to apply software issues to maritime topics. She serves a member of the Safety of Navigation committees of the International Maritime Organization and e-NAV committee of International Association of Lighthouse Authorities.

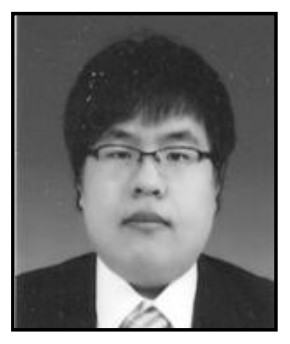

Hyoseung Kim, He is studying in master's degree at Korea Maritime and Ocean University. He is interested in web-services and software developments related to maritime safety information. $\mathrm{He}$ is also interested in studying the S-100 standard and related computing techniques.

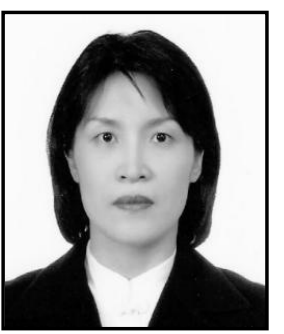

Hye-young Kim She received the B.Sc. degree and M.S. degree in Computer Science'from the Sookmyung women's University in South Korea and Ph.D. degree in Computer Science and Engineering from the Rorea University of South Korea in February 2005. Currently, she works at Hongik University of South Korea as an associate professor. Her research Interests include traffic modeling and location management in mobile network and wireless network for mobile game or multimedia service. 